\title{
ML estimation of timing, integer frequency and primary sequence index in LTE systems
}

\author{
Michele Morelli and Marco Moretti \\ Dipartimento di Ingegneria dell'Informazione, Università di Pisa \\ E-mail: \{michele.morelli,marco.moretti\}@iet.unipi.it
}

\begin{abstract}
This paper addresses the problem of maximum likelihood (ML) estimation of slot timing, integer carrier frequency offset and primary sequence index for the downlink of Long Term Evolution (LTE) systems. The proposed algorithm is designed to exploit the knowledge of the pilot Zadoff-Chu sequence embedded in the primary synchronization signal (PSS). The estimation process is affected by the presence of a large set of nuisance parameters, which need to be estimated jointly with the parameters of interest. As a consequence, the exact ML solution is extremely complex and we have developed a suboptimal algorithm designed to provide a good balance between estimation accuracy and complexity. In particular, a key finding is a reduced-rank representation for the frequency response of the channel, which is required by the ML estimator but is not available at receiver prior to having acquired synchronization. Compared to existing alternatives, the resulting scheme exhibits improved accuracy in the estimation of all three parameters of interest.
\end{abstract}

Index Terms-LTE, synchronization, cell search, primary synchronization signal.

\section{INTRODUCTION}

When registering on the LTE network, the user equipment (UE) activates a cell search procedure to acquire symbol timing, frame timing, carrier frequency offset and cell ID information.

In particular, the UE needs first to identify the host cell identity. To perform this task, the UE receiver needs to achieve frequency and timing synchronization.

Frequency synchronization is necessary to recover and correct the frequency error due to the local oscillator instability. In OFDM systems, the frequency error, normalized to subcarrier distance, is the sum of two terms: a fractional frequency offset (FFO), which destroys the orthogonality between subcarriers, and an integer frequency offset (IFO), which causes a circular shift of the subcarrier positions leading to a complete loss of the transmitted data.

To achieve full timing synchronization, in a LTE system the receiver first needs to acquire symbol synchronization, which establishes the beginning of the OFDM block so that the cyclic prefix $(\mathrm{CP})$ can be removed and the discrete Fourier transform (DFT) of the received samples can be computed, then slot synchronization that establishes the position of a symbol within a slot and eventually frame synchronization.

Accordingly, the synchronization procedure follows three separate steps:

1) In the time domain, the receiver performs FFO and symbol synchronization. By exploiting the presence of the
$\mathrm{CP}$, it has been shown [1] that the FFO can be estimated and corrected and that a coarse block timing estimate is available so that the frequency-domain received samples of each OFDM block can be computed without sustaining any relevant inter-block interference (IBI) .

2) In the frequency domain IFO is estimated and corrected, slot synchronization is acquired and partial information on cell identity based on the index of the pilot sequence is gathered by exploiting the pilot information contained in the primary synchronization signal (PSS).

3) In the frequency domain, frame synchronization and full cell identity is obtained by exploiting the pilot information of the secondary synchronization signal (SSS).

This paper deals with synchronization issues relative to the second step: slot synchronization, IFO estimation and pilot sequence index estimation. These parameters are usually estimated jointly taking advantage of the correlation properties of the Zadoff-Chu sequences embedded in the PSS. In [2] is presented a cell search algorithm that estimates the slot timing and pilot sequence by looking for the maximum of the correlation of the received signal with tentative values of the reference pilot signal. The main limit of this approach is that it suffers from the frequency selectivity of the channel that tends to destroy the correlation properties of the ZadoffChu pilot sequences. Other works address the problem of the selectivity of the propagation channel with ad hoc heuristics. In [3] and [4] the dependence on the frequency selective channel is removed by assuming that adjacent subcarriers have approximately the same propagation channel. Based on this assumption, estimation is performed on the signal obtained by multiplying one subcarrier with the conjugate complex of the next. Following the same line of reasoning, the algorithm in [5] computes the correlation between the received signal and the reference signal by performing several partial summation over sets of subcarriers with an approximately equal channel. In this paper we derive a rigorous maximum likelihood approach based on a reduced rank representation of the propagation channel, which is treated as a nuisance parameter. The results presented in this paper show that the ML estimator outperforms all the other schemes.

\section{SignAL MODEL}

\section{A. LTE frame structure}

We consider the downlink of a FDD LTE system. Data are transmitted in $10 \mathrm{~ms}$ radio frames, each radio frame consists 
of ten $1 \mathrm{~ms}$ sub-frames, and each sub-frame is organized in two consecutive $0.5 \mathrm{~ms}$ slots. Every slot is composed by six or seven OFDM blocks depending on the length of the cyclic prefix (CP).

As shown in Fig. 1, to enable cell search and synchronization, every ten slots (i.e. twice per frame) the PSS and the SSS are embedded in the transmitted signal. There are 504 physical-layer cell identities, divided into 168 groups of three. The identity of each group is indicated by one of the 168 possible SSS, while the cell identity within a single group is given by the index of the PSS. The two sequences, both generated in the frequency domain, occupy a specific position in the LTE time-frequency grid. The PSS is transmitted on the central 73 subcarriers of the last OFDM block of the first and eleventh slots of each radio frame and the SSS is transmitted over the same subcarriers in the block immediately preceding the PSS. The PSS is composed by eleven null symbols (five null symbols at both sequence's boundaries and one null symbol in correspondence to the d.c. subcarrier), and by 62 pilot symbols belonging to one of three different Zadhoff-Chu sequences $\left\{z c_{u}(n)\right\}=e^{-j \pi u\left(n^{2}+63 n+110\right) / 63}$, $\left(n \in \mathcal{I}_{p}=\{n \in \mathbb{Z}:|n| \leq 31\right.$ and $\left.n \neq 0\}\right)$, whose index $u \in \mathcal{J}_{U}=\{25,29,34\}$ provides the cell identity.

\section{B. OFDM signal}

At the receiver, the incoming signal is down-converted to baseband, sampled with period $T_{s}$ and converted in the frequency domain through an $N$-point DFT operation. Let us suppose that the PSS signal is present on the $q$ th block and let $X_{k}(n)(|n| \leq 36)$ be an element of the DFT of the $k$ th received block, $k \in \mathcal{J}_{Q}=\left\{1, \ldots, N_{Q}\right\}$. If $k \neq q$, we model $X_{k}(n)$ as a zero mean Gaussian variable with power $\sigma_{k}^{2}=\mathrm{E}\left\{\left|X_{k}(n)\right|^{2}\right\}$, if $k=q$ then the frequency domain samples of the received block can be written as

$$
X_{q}(n)=H_{q}(n-\nu) a_{u}(n-\nu)+w_{q}(n)
$$

where $a_{u}(n)=z c_{u}(n)$ if $n \in \mathcal{I}_{p}$ and zero otherwise, $w_{q}(n)$ is a disturbance terms that accounts for white Gaussian noise with power $\sigma_{w}^{2}=\mathrm{E}\left\{w_{q}(n)^{2}\right\}, \nu$ is the IFO and $H_{q}(n)$ is the channel frequency response that incorporates also the phase shift $e^{j 2 \pi \nu q N_{g} / N}$ that is accumulated at each block over the $N_{g}$ samples of the CP due to the IFO.

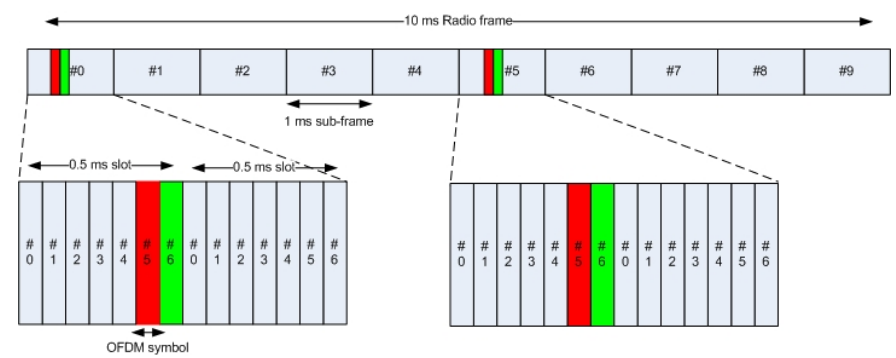

Fig. 1. Position of PSS (green) and SSS (red) in the LTE downlink frame.

\section{ML ESTIMATION OF $q, u$ AND $\nu$}

Since the PPS is transmitted twice per frame, we observe the $N_{Q}$ consecutive OFDM blocks composing a half frame to reveal the presence of the PSS. Our goal is the estimation of the integer frequency offset $\nu \in \mathcal{J}_{\nu}$, the block index $q \in \mathcal{J}_{Q}$ and the PSS sequence index $u \in \mathcal{J}_{U}$ based on the received frequency domain vector $\mathbf{X}=\left\{X_{k}(n) ;|n| \leq 36, k \in \mathcal{J}_{Q}\right\}$. The samples $X_{k}(n)$ are statistically independent and Gaussian distributed with zero mean and variance $\sigma_{k}^{2}$ if $k \neq q$ and with mean $H_{q}(n-\nu) a_{u}(n-\nu)$ and variance $\sigma_{w}^{2}$ if $k=q$. Hence, in presence of the unknown parameters $\tilde{\mathbf{H}}_{\tilde{q}}=\left\{\tilde{H}_{\tilde{q}}(n) ;|n| \leq\right.$ $36\}, \tilde{\sigma}_{w}^{2}, \tilde{\sigma}_{k}^{2}$ the ML function takes the form

$$
\begin{aligned}
& p\left(\mathbf{X} \mid \tilde{\varphi}, \tilde{\mathbf{H}}_{\tilde{q}}, \tilde{\sigma}_{w}^{2}, \tilde{\sigma}_{k}^{2}\right)=\prod_{\substack{k=1 \\
k \neq \tilde{q}}}^{N_{Q}} \frac{1}{\left(\pi \tilde{\sigma}_{k}^{2}\right)^{73}} e^{-\frac{\sum_{n=-36}^{36}\left|X_{k}(n)\right|^{2}}{\tilde{\sigma}_{k}^{2}}} \\
& \times \frac{1}{\left(\pi \tilde{\sigma}_{w}^{2}\right)^{73}} e^{-\frac{\sum_{n=-36}^{36}\left|\tilde{\tilde{q}}_{\tilde{q}}(n)-\tilde{H}_{\tilde{q}}(n-\tilde{\nu}) a \tilde{u}(n-\tilde{\nu})\right|^{2}}{\tilde{\sigma}_{w}^{2}}}
\end{aligned}
$$

where the vector $\tilde{\varphi}=[\tilde{q}, \tilde{u}, \tilde{\nu}]$ collects the unknown estimation parameters.

The joint ML estimate of the unknown parameters is the location where $p\left(\mathbf{X} \mid \tilde{\boldsymbol{\varphi}}, \tilde{\mathbf{H}}_{\tilde{q}}, \tilde{\sigma}_{w}^{2}, \tilde{\sigma}_{k}^{2}\right)$ achieves its global maximum, which can be equivalently found by looking for the maximum of the log-likelihood function (LLF) $\Lambda\left(\tilde{\boldsymbol{\varphi}} ; \tilde{\mathbf{H}}_{\tilde{q}}, \tilde{\sigma}_{w}^{2}, \tilde{\sigma}_{k}^{2}\right)$ given by

$$
\begin{aligned}
& \Lambda\left(\tilde{\boldsymbol{\varphi}} ; \tilde{\mathbf{H}}_{\tilde{q}}, \tilde{\sigma}_{w}^{2}, \tilde{\sigma}_{k}^{2}\right)= \\
& \sum_{\substack{k=1 \\
k \neq \tilde{q}}}^{N_{Q}}\left(-73 \log \left(\pi \tilde{\sigma}_{k}^{2}\right)-\frac{\sum_{n=-36}^{36}\left|X_{k}(n)\right|^{2}}{\tilde{\sigma}_{k}^{2}}\right) \\
& -73 \log \left(\pi \tilde{\sigma}_{w}^{2}\right)-\frac{\sum_{n=-36}^{36}\left|w\left(n ; \tilde{\boldsymbol{\varphi}}, \tilde{\mathbf{H}}_{\tilde{q}}\right)\right|^{2}}{\tilde{\sigma}_{w}^{2}}
\end{aligned}
$$

where we have defined

$$
w\left(n ; \tilde{\boldsymbol{\varphi}}, \tilde{\mathbf{H}}_{\tilde{q}}\right)=X_{\tilde{q}}(n)-\tilde{H}_{\tilde{q}}(n-\tilde{\nu}) a_{\tilde{u}}(n-\tilde{\nu}) .
$$

Maximizing $\Lambda\left(\tilde{\boldsymbol{\varphi}} ; \tilde{\mathbf{H}}_{\tilde{q}}, \tilde{\sigma}_{w}^{2}, \tilde{\sigma}_{k}^{2}\right)$ with respect to $\tilde{\sigma}_{k}^{2}$ and $\tilde{\sigma}_{w}^{2}$ yields

$$
\hat{\sigma}_{k}^{2}=\frac{1}{73} \sum_{n=-36}^{36}\left|X_{k}(n)\right|^{2}
$$

and

$$
\hat{\sigma}_{w}^{2}=\frac{1}{73} \sum_{n=-36}^{36}\left|w\left(n ; \tilde{\boldsymbol{\varphi}}, \tilde{\mathbf{H}}_{\tilde{q}}\right)\right|^{2}
$$

By replacing the values of $\tilde{\sigma}_{k}^{2}$ and $\tilde{\sigma}_{w}^{2}$ found in (5) and (6) and adding and subtracting the term $73 \log \left(\frac{\pi}{73} \sum_{n=-36}^{36}\left|X_{q}(n)\right|^{2}\right)$, the LLF (3) can be rewritten 
as

$$
\begin{aligned}
& \Lambda\left(\tilde{\boldsymbol{\varphi}} ; \tilde{\mathbf{H}}_{\tilde{q}}\right)=-73 N_{Q}-73 \sum_{k=1}^{N_{Q}} \log \left(\frac{\pi}{73} \sum_{n=-36}^{36}\left|X_{k}(n)\right|^{2}\right) \\
& +73 \log \left(\frac{\pi}{73} \sum_{n=-36}^{36}\left|X_{q}(n)\right|^{2}\right) \\
& -73 \log \left(\frac{\pi}{73} \sum_{n=-36}^{36}\left|w\left(n ; \tilde{\boldsymbol{\varphi}}, \tilde{\mathbf{H}}_{\tilde{q}}\right)\right|^{2}\right)
\end{aligned}
$$

Skipping irrelevant additive terms independent of the optimization variables and exploiting the monotonicity of the log operator, we obtain that the maximum of LLF is found by maximizing the concentrated $\operatorname{LLF} \Phi\left(\tilde{\varphi} ; \tilde{\mathbf{H}}_{\tilde{q}}\right)$

$$
\Phi\left(\tilde{\boldsymbol{\varphi}} ; \tilde{\mathbf{H}}_{\tilde{q}}\right)=-\frac{\sum_{n=-36}^{36}\left|w\left(n ; \tilde{\boldsymbol{\varphi}}, \tilde{\mathbf{H}}_{\tilde{q}}\right)\right|^{2}}{\sum_{n=-36}^{36}\left|X_{\tilde{q}}(n)\right|^{2}}
$$

Moreover, making a change of the indexing variable ( $n-$ $\nu \rightarrow n$ ) into (4), taking into account that the values of the pilot sequence $a_{u}$ are zero in certain specific positions and dropping immaterial factors in (8), one obtains a new function $\Phi_{1}\left(\tilde{\varphi} ; \tilde{\mathbf{H}}_{\tilde{q}}\right)$, whose maximization yields the ML estimate of the unknown parameters

$$
\begin{aligned}
& \Phi_{1}\left(\tilde{\boldsymbol{\varphi}} ; \tilde{\mathbf{H}}_{\tilde{q}}\right)= \\
& \frac{2 \mathbb{R e}\left\{\sum_{\substack{n=-31 \\
n \neq 0}}^{31} \tilde{H}_{\tilde{q}}^{*}(n) Z_{\tilde{q}}(n ; \tilde{u}, \tilde{\nu})\right\}-\sum_{\substack{n=-31 \\
n \neq 0}}^{31}\left|\tilde{H}_{\tilde{q}}(n)\right|^{2}}{\sum_{n=-36}^{36}\left|X_{\tilde{q}}(n)\right|^{2}}
\end{aligned}
$$

where it is

$$
Z_{\tilde{q}}(n ; \tilde{u}, \tilde{\nu})=X_{\tilde{q}}(n+\tilde{\nu}) a_{\tilde{u}}^{*}(n) \quad n \in \mathcal{I}_{p}
$$

In the following, with a slight abuse of notation, we redefine the vector of the channel coefficients as $\mathbf{H}_{q}=\left\{H_{q}(n) ; n \in\right.$ $\mathcal{I}_{p}$, i.e., considering only those subcarriers where the PSS is different from zero. Let us assume that the channel samples are correlated so that $\mathbf{H}_{q}$ can be expressed as a linear combination of the terms of the $P$-dimensional (with $P \ll 62$ ) orthonormal basis $\left\{\mathbf{b}_{1}, \ldots, \mathbf{b}_{P}\right\}$ as

$$
\mathbf{H}_{q}=\mathbf{B} \xi
$$

where $\mathbf{B}=\left[\mathbf{b}_{1}, \ldots, \mathbf{b}_{P}\right]$ is the $62 \times P$ matrix collecting the basis vectors and $\boldsymbol{\xi}=\left[\xi_{1}, \xi_{2}, \ldots, \xi_{P}\right]^{T}$ is the $P$-dimensional vector of the expansion coefficients on the basis $\mathbf{B}$.

Upon defining the vector $\mathbf{X}_{\tilde{q}}(\tilde{\nu})=\left\{X_{\tilde{q}}(n+\tilde{\nu}) ; n \in \mathcal{I}_{p}\right\}$ and the matrix $\mathbf{A}_{\tilde{u}}=\operatorname{diag}\left\{a_{\tilde{u}}(n) ; n \in \mathcal{I}_{p}\right\}$, the concentrated LLF can be rewritten in a more compact form as

$$
\Phi_{1}(\tilde{\boldsymbol{\varphi}} ; \tilde{\boldsymbol{\xi}})=\frac{2 \mathbb{R e}\left\{\mathbf{X}_{\tilde{q}}(\tilde{\nu})^{H} \mathbf{A}_{\tilde{u}} \mathbf{B} \tilde{\boldsymbol{\xi}}\right\}-\tilde{\boldsymbol{\xi}}^{H} \mathbf{B}^{H} \mathbf{B} \tilde{\boldsymbol{\xi}}}{\sum_{n=-36}^{36}\left|X_{\tilde{q}}(n)\right|^{2}}
$$

By differentiating $\Phi_{1}(\tilde{\boldsymbol{\varphi}} ; \tilde{\boldsymbol{\xi}})$ with respect to $\tilde{\boldsymbol{\xi}}$ and setting the results to zero, one obtains the ML estimate of $\boldsymbol{\xi}$

$$
\hat{\boldsymbol{\xi}}=\left(\mathbf{B}^{H} \mathbf{B}\right)^{-1} \mathbf{B}^{H} \mathbf{A}_{\tilde{u}}^{H} \mathbf{X}_{\tilde{q}}(\tilde{\nu})
$$

By substituting in (12) the value for $\boldsymbol{\xi}$ found in (13), the new concentrated LLF takes the form

$$
\Phi_{2}(\tilde{\varphi})=\frac{\mathbf{Z}_{\tilde{q}}(\tilde{u}, \tilde{\nu})^{H} \mathbf{G} \mathbf{Z}_{\tilde{q}}(\tilde{u}, \tilde{\nu})}{\sum_{n=-36}^{36}\left|X_{\tilde{q}}(n)\right|^{2}}
$$

where $\mathbf{G}=\mathbf{B}\left(\mathbf{B}^{H} \mathbf{B}\right)^{-1} \mathbf{B}^{H}$ and $\mathbf{Z}_{\tilde{q}}(\tilde{u}, \tilde{\nu})=\mathbf{A}_{\tilde{u}}^{H} \mathbf{X}_{\tilde{q}}(\tilde{\nu})$ is the vector stacking the values of $Z_{\tilde{q}}(n ; \tilde{u}, \tilde{\nu})$.

To proceed further, we need to find an appropriate expansion basis $\mathbf{B}$ for $\mathbf{H}_{q}$.

\section{A. A reduced-rank base for $\mathbf{H}_{q}$}

Letting $\mathbf{h}=[h(0), h(1), \ldots, h(L-1)]$ be the length $L$ impulse response of the propagation channel, the channel frequency response $\mathbf{H}$ can be factorized as

$$
\mathbf{H}=\mathbf{F h}
$$

where $\mathbf{F}$ is a $62 \times L$ matrix, whose elements are

$$
[\mathbf{F}]_{n, \ell}=e^{-j 2 \pi n \ell / N} \quad n \in \mathcal{I}_{p} ; \ell=0,1, \ldots, L-1
$$

so that it is $\mathbf{G}=\mathbf{F}\left(\mathbf{F}^{H} \mathbf{F}\right)^{-1} \mathbf{F}^{H}$. In many cases it might be $L \gg 62$ and the matrix $\mathbf{F}^{H} \mathbf{F}$ not invertible. Thus, to avoid this problem and to pursue in general a low complexity approach, we aim at finding a rank-reduced expansion employing a base $\mathbf{F}_{p}$ of dimension $P \ll L$ for the vector, so that it is $\mathbf{H}_{p}=\mathbf{F}_{p} \boldsymbol{\xi}$ and $\mathbf{H}_{p} \approx \mathbf{H}$.

Our objective is to find the vector $\mathbf{H}_{p}$ that, given the dimension $P$ of the expansion basis, minimises the mean square error (MSE), i.e.

$$
\begin{gathered}
\min _{\mathbf{F}_{p}, \boldsymbol{\xi}} \mathrm{E}\left\{\left\|\mathbf{H}-\mathbf{F}_{p} \boldsymbol{\xi}\right\|^{2}\right\} \\
\text { subject to } \\
\operatorname{rank} \mathbf{F}_{p}=P
\end{gathered}
$$

First of all, we observe that, given the matrix $\mathbf{F}_{p}$, the vector $\boldsymbol{\xi}$ that minimises the metric $\left\|\mathbf{H}-\mathbf{F}_{p} \boldsymbol{\xi}\right\|^{2}$ is the least square (LS) estimator

$$
\boldsymbol{\xi}_{L S}=\left(\mathbf{F}_{p}^{H} \mathbf{F}_{p}\right)^{-1} \mathbf{F}_{p}^{H} \mathbf{H}
$$

so that, consequently, $\mathbf{H}_{p}$ can be written as

$$
\mathbf{H}_{p}=\mathbf{F}_{p}\left(\mathbf{F}_{p}^{H} \mathbf{F}_{p}\right)^{-1} \mathbf{F}_{p}^{H} \mathbf{H}
$$

Thus, we need to find the matrix $\mathbf{F}_{p}$ that minimises $\gamma\left(\mathbf{F}_{p}\right)=$ $\mathrm{E}\left\{\left\|\mathbf{H}-\mathbf{F}_{p} \boldsymbol{\xi}_{L S}\right\|^{2}\right\}$. By replacing (15) in (19), one obtains $\mathbf{H}-\mathbf{F}_{p} \boldsymbol{\xi}_{L S}=\left(\mathbf{I}_{62}-\mathbf{F}_{p}\left(\mathbf{F}_{p}^{H} \mathbf{F}_{p}\right)^{-1} \mathbf{F}_{p}^{H}\right) \mathbf{F h}$, and the MMSE takes the form

$$
\operatorname{MMSE}\left(\mathbf{F}_{p}\right)=\operatorname{tr}\left\{\left(\mathbf{I}_{62}-\mathbf{F}_{p}\left(\mathbf{F}_{p}^{H} \mathbf{F}_{p}\right)^{-1} \mathbf{F}_{p}^{H}\right) \mathbf{F} \mathbf{C}_{h} \mathbf{F}^{H}\right\}
$$

where $\mathbf{C}_{h}=\mathrm{E}\left\{\mathbf{h} \mathbf{h}^{H}\right\}$ is the channel covariance matrix. 
After neglecting a constant term, minimizing (20) is tantamount to maximise the metric $\gamma\left(\mathbf{F}_{p}\right)$

$$
\gamma\left(\mathbf{F}_{p}\right)=\operatorname{tr}\left\{\mathbf{F}_{p}\left(\mathbf{F}_{p}^{H} \mathbf{F}_{p}\right)^{-1} \mathbf{F}_{p}^{H} \mathbf{F} \mathbf{C}_{h} \mathbf{F}^{H}\right\}
$$

Let $\mathbf{F}_{p}=\left[\mathbf{U}_{p}^{(1)}, \mathbf{U}_{p}^{(0)}\right] \boldsymbol{\Sigma}_{p} \mathbf{V}_{p}^{H}$ be the singular-value decomposition (SVD) of $\mathbf{F}_{p}$, where $\mathbf{U}_{p}^{(1)}$ are the $P$ right eigenvectors associated to the positive eigenvalues of $\mathbf{F}_{p}$ and $\mathbf{U}_{p}^{(0)}$ are the $62-P$ right eigenvectors associated to null eigenvalues. By simple algebraic manipulation, the metric $\gamma\left(\mathbf{F}_{p}\right)$ can be written as

$$
\gamma\left(\mathbf{F}_{p}\right)=\operatorname{tr}\left\{\mathbf{U}_{p}^{(1) H} \mathbf{F} \mathbf{C}_{h} \mathbf{F}^{H} \mathbf{U}_{p}^{(1)}\right\} .
$$

The metric $\gamma\left(\mathbf{F}_{p}\right)$ depends only on $\mathbf{U}_{p}^{(1)}$ and the maximisation of (22) with respect to $\mathbf{U}_{p}^{(1)}$ is a well-known optimization problem, whose solution is obtained by choosing the $P$ eigenvectors associated to the $P$ largest eigenvalues of the matrix $\mathbf{F C}_{h} \mathbf{F}^{H}$ as columns of $\mathbf{U}_{p}^{(1)}$. Therefore, the optimal solution of (21) does not depend on $\mathbf{U}_{p}^{(0)}, \boldsymbol{\Sigma}_{p}$ and $\mathbf{V}_{p}$ and the last two matrices can be arbitrarily chosen so that $\mathbf{F}_{p}=\mathbf{U}_{p}^{(1)}$ and $\boldsymbol{\xi}=\left(\mathbf{F}_{p}^{H} \mathbf{F}_{p}\right)^{-1} \mathbf{F}_{p}^{H} \mathbf{H}$ is a solution of (17).

In most cases, the receiver does not possess any knowledge on the matrix $\mathbf{C}_{h}$ and a low-complexity off-the-shelf solution consists in setting $\mathbf{C}_{h}=\mathbf{I}_{L}$ with an arbitrary value of $L$. In this case, the matrix $\mathbf{U}_{p}^{(1)}$ is found by simply collecting the $P$ eigenvectors corresponding to the $P$ largest eigenvalues of $\mathbf{F F}^{H}$.

Replacing $\mathbf{B}$ with $\mathbf{F}_{p}$ in (14) and exploiting the fact that, by construction, it is $\mathbf{F}_{p}^{H} \mathbf{F}_{p}=\mathbf{I}_{P}$ yields

$$
\Phi_{2}(\tilde{\boldsymbol{\varphi}})=\frac{\left\|\mathbf{F}_{p}^{H} \mathbf{Z}_{\tilde{q}}(\tilde{u}, \tilde{\nu})\right\|^{2}}{\sum_{n=-36}^{36}\left|X_{\tilde{q}}(n)\right|^{2}}
$$

and the parameters of interest are estimated by maximizing $\Phi_{2}(\tilde{\varphi})$

$$
\hat{q}, \hat{u}, \hat{\nu}=\arg \max _{\tilde{q}, \tilde{u}, \tilde{\nu}} \Phi_{2}(\tilde{q}, \tilde{u}, \tilde{\nu})
$$

\section{REVIEW OF EXISTING CELL SEARCH AND SYNCHRONISATION SCHEMES FOR LTE}

We present here a review of the most common algorithms employed in recent literature to perform cell search and synchronisation in LTE systems. To place the various schemes in an adequate framework and perform a fair comparison, some algorithms have been slightly modified.

1) Conventional estimator $(C E)$ : The work in [2] exploits the autocorrelation properties of the $\mathrm{ZC}$ sequences. The vector of unknown parameters $\varphi$ is estimated by searching the maximum of the correlation of the received signal with the pilot sequence $a_{u}(n)$

$$
\hat{q}, \hat{u}, \hat{\nu}=\arg \max _{\tilde{q}, \tilde{u}, \tilde{\nu}} \frac{\left|\sum_{n \in \mathcal{I}_{P}} Z_{\tilde{q}}(n ; \tilde{u}, \tilde{\nu})\right|^{2}}{\sum_{n \in \mathcal{I}_{P}}\left|X_{\tilde{q}}(n+\tilde{\nu})\right|^{2}}
$$

2) Partially coherent estimator (PCE): The main problem of the method in (25) is represented by the selectivity of the propagation channel, which tends to decorrelate the terms in the sum at the numerator and to destroy the properties of the $\mathrm{ZC}$ sequences. To partially solve this impairment, the authors in [5] replace the sum in the numerator of (25) with $63 / M$ partial summations, each adding together the contribution of $M$ contiguous subcarriers.

$$
\hat{q}, \hat{u}, \hat{\nu}=\arg \max _{\tilde{q}, \tilde{u}, \tilde{\nu}} \frac{\sum_{m=1}^{63 / M}\left|\sum_{n=-31+(m-1) M}^{-31+m M-1} Z_{\tilde{q}}(n ; \tilde{u}, \tilde{\nu})\right|^{2}}{\sum_{n \in \mathcal{I}_{P}}\left|X_{\tilde{q}}(n+\tilde{\nu})\right|^{2}}
$$

where the optimal value of $M$ depends on the channel coherence bandwidth.

3) Differential estimator $(D E)$ : The differential estimator proposed in [3] attenuates the dependence on the frequency selectivity of the channel by multiplying adjacent frequency received samples so that $H_{q}(n) H_{q}(n-1)^{*}$ can be approximated as $\left|H_{q}(n)\right|^{2}$. Hence, the triplet $(q, u, \nu)$ is estimated as

$$
\hat{q}, \hat{u}, \hat{\nu}=\arg \max _{\tilde{q}, \tilde{u}, \tilde{\nu}} \frac{\Re \mathrm{e}\left\{\sum_{n \in \mathcal{I}_{P} /\{1\}} Z_{\tilde{q}}(n ; \tilde{u}, \tilde{\nu}) Z_{\tilde{q}}(n-1 ; \tilde{u}, \tilde{\nu})^{*}\right\}}{\sum_{n \in \mathcal{I}_{P}}\left|X_{\tilde{q}}(n+\tilde{\nu})\right|^{2}}
$$

\section{NUMERICAL RESULTS}

In this section we compare the performance of the discussed synchronization algorithms deployed in a LTE system compliant with the 3GPP specifications [6]. The signal bandwidth is $20 \mathrm{MHz}, \mathrm{CP}$ length is normal, FFT size is 2048 and sampling rate is 30.72 megasample/s.

Results are obtained by employing the power delay profile of the extended typical urban (ETU) channel [7] summarized in Table I. The gains of the paths are modelled as independent circularly symmetric Gaussian random variables with zeromean (Rayleigh fading).

TABLE I

ETU CHANNEL POWER PROFILE

\begin{tabular}{||c|c||}
\hline Excess tap delay $(\mathrm{ns})$ & Relative power $(\mathrm{dB})$ \\
\hline \hline 0 & -1.0 \\
\hline 50 & -1.0 \\
\hline 120 & -1.0 \\
\hline 200 & 0.0 \\
\hline 230 & 0.0 \\
\hline 500 & 0.0 \\
\hline 1600 & -3.0 \\
\hline 2300 & -5.0 \\
\hline 5000 & -7.0 \\
\hline
\end{tabular}

Considered that one PSS is transmitted every 10 slots and that there are seven (normal CP) OFDM symbols per slot, it is $N_{Q}=70$. Assuming that the stability of commercial oscillators for mobile applications is in the range of 10 
parts per million (ppm) and the highest carrier frequency is $4 \mathrm{GHz}$, the largest possible frequency error is around 40 $\mathrm{kHz}$, which approximately corresponds to 2.66 the subcarrier spacing and, accordingly, the residual IFO takes value in the set $\mathcal{J}_{\nu}=\{0, \pm 1, \pm 2, \pm 3\}$. Moreover, there are three different Zadoff-Chu sequences, so that the overall search space for the synchronization algorithm consists of $60 \times 7 \times 3$ search values.

The performance of the joint estimator of the slot timing, IFO and pilot sequence index is assessed in terms of the error rate computed for each parameter of interest. For the ML estimator (MLE) the channel length has been arbitrarily set to $L=200$ samples corresponding to a maximum channel impulse response duration of approximately $6.5 \mu \mathrm{s}$ and the parameter $P=9$. For the PCE we have chosen $M=7$ as the value that maximizes its performance.

Fig. 2 shows the slot timing error as function of the SNR. As it is seen, MLE outperforms all other estimators. The DE is second best but loses almost two $\mathrm{dB}$ in comparison to MLE. Since the frequency selectivity of the channel is large, CE performs rather poorly and exhibits a performance floor already for low SNR values.

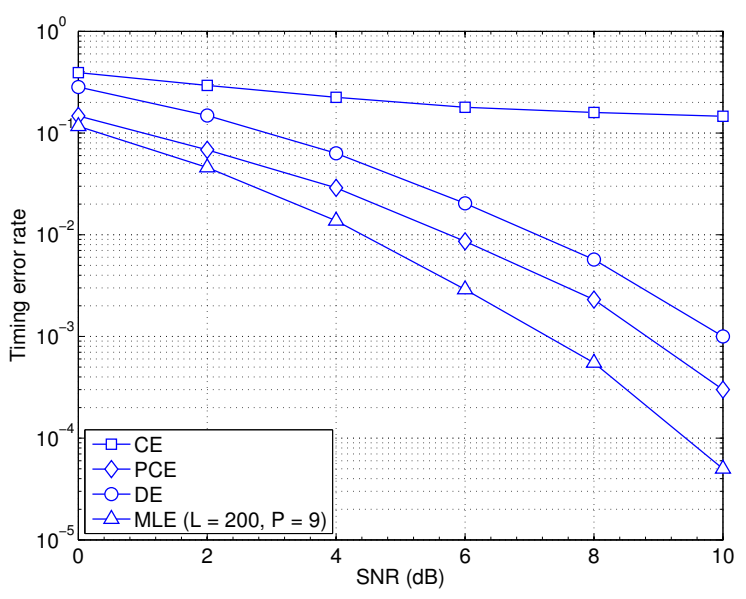

Fig. 2. Timing error rate as function of the SNR.

Fig. 3 shows the results for the error rate of the Zadoff-Chu sequence index estimation. The trend is similar to that of Fig. 1 with MLE outperforming all other estimation schemes.

Fig. 4 plots the IFO error rate and in this case the gain of MLE over all other schemes is much more evident than for the two other parameters of interest.

\section{Conclusions}

This paper has presented a maximum likelihood algorithm for joint estimation of slot timing, integer carrier frequency offset and primary sequence index for the downlink of Long Term Evolution systems. The proposed algorithm exploits the knowledge of the pilot Zadoff-Chu sequence transmitted in the primary synchronization signal. The main novelty of the proposed scheme consists in introducing a reduced-rank representation of the channel frequency response, which strikes a good balance between estimation performance and implementation

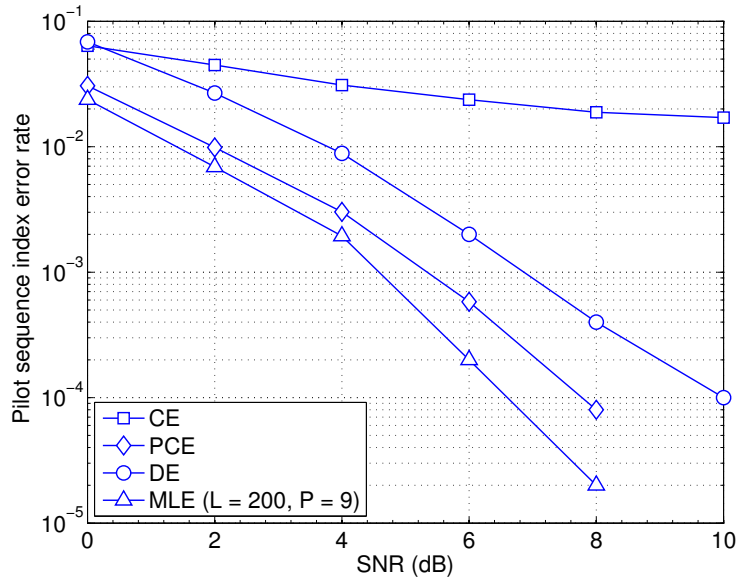

Fig. 3. Pilot sequence index error rate as function of the SNR.

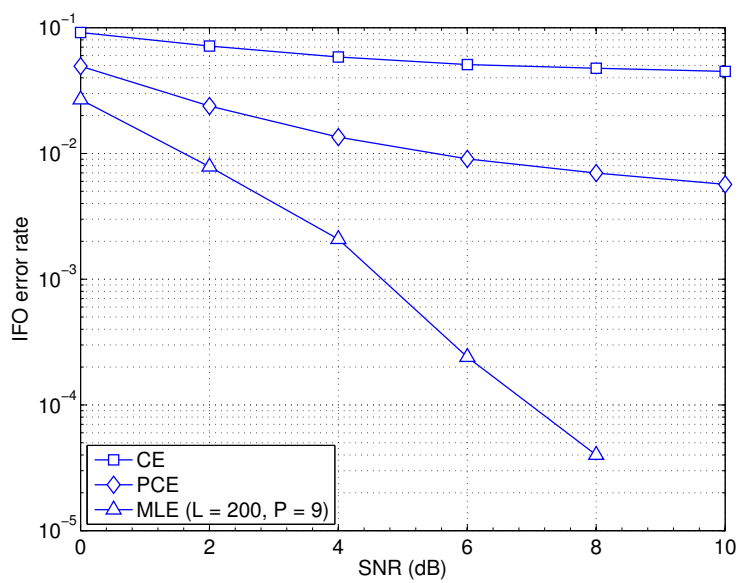

Fig. 4. IFO error rate as function of the SNR.

load. Simulation results show that the proposed estimator is able to clearly outperform all other relevant algorithms present in the literature.

\section{REFERENCES}

[1] J.-J. van de Beek, P. Borjesson, M.-L. Boucheret, D. Landstrom, J. Arenas, P. Odling, C. Ostberg, M. Wahlqvist, and S. Wilson, "A time and frequency synchronization scheme for multiuser ofdm," Selected Areas in Communications, IEEE Journal on, vol. 17, no. 11, pp. 1900-1914, Nov. 1999.

[2] K. Manolakis, D. Gutierrez Estevez, V. Jungnickel, W. Xu, and C. Drewes, "A closed concept for synchronization and cell search in 3GPP LTE systems," in Proc. of IEEE WCNC, 2009, pp. 1-6.

[3] P. Tsai and H. Chang, "A new cell search scheme in 3GPP long term evolution downlink OFDMA systems," in Proc. of IEEE WCSP, 2009.

[4] S. Su, Y. Lin, and Y. Fan, "Joint sector identity and integer part of carrier frequency offset detection by phase-difference in long term evolution cell search process," IET Communications, vol. 7, no. 10, 2013.

[5] L.-C. Wung, Y.-C. Lin, Y.-J. Fan, and S.-L. Su, "A robust scheme in downlink synchronization and initial cell search for 3GPP LTE system," in Proc. 6th ISWPC, Feb 2011, pp. 1-6.

[6] 3GPP, "TS 36.211; Evolved Universal Terrestrial Radio Access (EUTRA); Physical channels and modulation," Tech. Rep., 2009.

[7] — "TS 36.101; Technical Specification Group Radio Access Network; Evolved Universal Terrestrial Radio Access (E-UTRA); User Equipment (UE) radio transmission and reception," Tech. Rep., 2009. 